\title{
Clima Laboral en Empresas Ecuatorianas fabricantes de Carrocerías: Caso Canfac
}

\section{Labor Climate in Ecuadorian Companies Manufacturers of Bodyworks: Case Canfac}

INFORMACIÓN DEL

\section{ARTÍCULO}

Fecha de recepción: 17 de Octubre de 2019.

Fecha de aceptación: 26 de Mayo de 2020 .

${ }^{1}$ Master of Arts en Psicología Industrial, Fairleigh Dickinson University. Docente e Investigador de la Pontificia Universidad Católica del Ecuador Sede Ambato PUCESA-Ecuador.

E-mail: spazmay@pucesa.edu.ec

Código ORCID:

https://orcid.org/0000-0001-7646-8070

2 Máster en Dirección, Universidad de Sancti Spiritus José Martí. Docente e Investigadora de la Pontificia Universidad Católica del Ecuador Sede Ambato PUCESA-Ecuador.

E-mail:dlima@pucesa.edu.ec

Código ORCID:

https://orcid.org/0000-0002-6554-1284

CITACIÓN: Pasmay Ramos, S.G., \& Lima Rojas, D. (2020). Clima Laboral en Empresas Ecuatorianas Fabricantes de Carrocerías: Caso CANFAC Podium, 37, 15-26.

doi:10.31095/podium.2020.37.2

\section{ENLACE DOI:}

http://dx.doi.org/10.31095/podium.202 0.37 .2

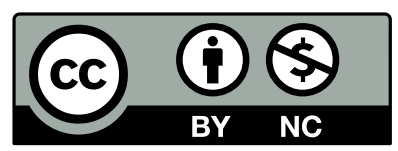

Segundo Gonzalo Pazmay Ramos ${ }^{1}$, Dayamy Lima Rojas ${ }^{2}$

\section{Resumen}

El objetivo de este estudio es describir el clima laboral en empresas ecuatorianas por medio de cinco dimensiones: condiciones de trabajo, compensaciones, comunicación, liderazgo, y motivación. El instrumento presentó un alfa de Cronbach de 0.85 , y se validó a través del análisis factorial. se aplicó a 478 personas de 12 empresas ecuatorianas. Los resultados señalaron que los encuestados se sienten motivados en el trabajo, existe una comunicación medianamente fluida, un estilo de liderazgo directivo -alto interés en la ejecución de la tarea y bajo en el relacionamiento-, las condiciones de trabajo y compensaciones son percibidas como satisfactorias. Se concluye que las medias aritméticas de las tres primeras dimensiones implican una respuesta de mediana frecuencia; y las de las dos últimas dimensiones una de mediana-alta frecuencia.

\section{Palabras Clave:}

Estudio descriptivo; clima laboral; comportamiento organizacional; desarrollo organizacional; psicología del trabajo; empresas ecuatorianas de producción.

Clasificación JEL: M10; M12; M14.

\begin{abstract}
The aim of this research is to describe the labor environment in Ecuadorian companies through five dimensions: working conditions, compensation, communication, leadership, and motivation; in Ecuadorian companies. The instrument registered a Cronbach's alpha of 0.85 , and it was validated through the factor analysis. It was applied to 478 people from 12 Ecuadorian companies. The results indicated that the respondents feel motivated at work, there is fairly fluid communication, a style of managerial leadership -high interest in the execution of the task and low in the relationship-, the working conditions and compensations are perceived as satisfactory. It is concluded that the arithmetic means of the first three dimensions imply a medium frequency response; and those of the last two dimensions a medium-high frequency.
\end{abstract}

\section{Keywords:}

Descriptive study; labor climate; organizational behavior; organizational development; work psychology; Ecuadorian production companies.

JEL Classification: M10; M12; M14. 


\section{Introducción}

Las empresas asociadas a la Cámara Nacional de Fabricantes de Carrocerías del Ecuador (CANFAC), atraviesan serias dificultades como: gestión administrativa clásica, inadecuado liderazgo, talento humano mal seleccionado, limitada capacitación del personal, ausencia de trabajo en equipo y deficiente comunicación, cuyas consecuencias han dado lugar a: incumplimiento de metas y objetivos, desmotivación del personal, alta rotación, baja productividad, escaso rendimiento y relaciones interpersonales conflictivas. En el Ecuador, y específicamente la provincia del Tungurahua se han realizado pocos estudios sobre clima laboral en las empresas productoras de carrocerías, consecuentemente carecen de herramientas propias de la realidad laboral local que les permita mejorar la gestión de los procesos organizacionales. Esta investigación constituye una contribución de valor para la literatura de clima organizacional en Ecuador, dados los pocos trabajos que han analizado el clima laboral en las empresas productoras locales y nacionales. Además, puede orientar a los directivos y/o consultores empresariales en las áreas a intervenir dentro de las organizaciones (Guízar, 2013).

Según el presidente de la CANFAC, la producción de carrocerías es uno de los pilares en la economía de Tungurahua, en esta provincia funcionan 19 empresas carroceras; allí se da empleo a cerca de 2 500 personas. La producción provincial cubre el $65 \%$ del mercado nacional (Jácome, 2017). A luz de estos datos, el presente estudio se vuelve relevante y de enorme importancia puesto que va a permitir conocer la dinámica de estos fenómenos; el impacto socioeconómico de este sector en la sociedad ecuatoriana es indiscutible, el conocer el clima organizacional que existe en estas empresas, permitirá mejorar el ambiente laboral.

El clima organizacional (CO) es uno de los conceptos determinantes para mejorar el desempeño laboral de las organizaciones, puesto que tiene incidencia en la productividad. Lamentablemente en muchos casos a los gerentes y directivos les ha tomado mucho tiempo darse cuenta de que los aspectos ligeros de los recursos humanos pueden tener una función decisiva en el éxito (o fracaso) de una organización (Furnham, 2006). El entender el CO de estas organizaciones, guiará intervenciones organizacionales para enriquecerlo.

\section{Revisión de literatura}

Los primeros estudios sobre clima laboral fueron realizados por Lewin (1988) y colaboradores en la década de los años treinta del siglo XX y éstos se centraban mayormente en la relación entre los líderes y sus seguidores, por ser un aspecto fundamental que influye en el clima organizacional. Autores como Likert (2007) y McGregor (1960) propusieron la utilización del concepto clima organizacional o clima laboral indistintamente, estaban interesados en la influencia de los directivos en la creación de un determinado clima en relación con sus subordinados, y analizaron cómo esto 
influía en los objetivos de la organización. En este sentido se podría considerar el estudio del clima como una fotografía de lo que está sucediendo en ese momento en la organización (Alcover de la Hera et al., Martínez, Rodríguez, y Domínguez, 2004).

La trascendencia de los estudios de CO está dada por el impacto de éste en la productividad, lo cual está intrínsecamente ligado al desempeño de los trabajadores, unido a la participación, motivación y un efectivo estilo de liderazgo (Cardona y Zambrano, 2014; Marchant, 2005).

Con relación a la participación, es importante poner énfasis en el individuo, sin soslayar la productividad, ya que las empresas en la que los empleados trabajan a gusto y tienen menos conflictos laborales son las que obtienen mejores resultados, en algunos casos hasta un $30 \%$ pueden crecer los beneficios (Silva, Santos, Rodríguez, y Hernando, 2008). En cuanto a la motivación, es prioridad tomar en cuenta las teorías motivacionales que impulsan a los individuos a la acción y en este sentido Davis y Newstrom (2003), afirman que un comportamiento organizacional eficiente es crear una motivación positiva, y por último adaptar el estilo de liderazgo para crear un ambiente adecuado en que los individuos trabajando en equipo logren metas y objetivos previamente establecidos. Así lo resaltan Flores, Vega, y Chávez (2015) cuando dicen que el $\mathrm{CO}$ es un punto sensible y difícil de cambiarlo, y se requiere que los directivos desarrollen capacidades estratégicas, técnicas, interpersonales y emocionales, para provocar un cambio organizacional.

Existe una diversidad de modelos y de instrumentos para medir el clima organizacional y que han sido aplicados en muchas organizaciones. Estudios para analizar el clima organizacional en el sector carrocero en el Ecuador son pocos, lo que ha motivado a realizar esta investigación. Cabe recalcar que se han efectuado investigaciones sobre $\mathrm{CO}$ en países como Cuba (Salazar, Guerrero, Machado, y Cañedo, 2009; Iglesias y Sánchez, 2015); Colombia (García, 2011; Echeverri y Cruz, 2014); Ecuador (Barzola-Zambrano, Barzola-Véliz, y Flores-Barzola, 2017); India (Bamel, Rangnekar, Stokes, y Rastogi, 2015); Perú (Gamboa-León, 2014); entre otros.

Romero (2008) considera que los elementos que conforman el clima son: salud, equidad, seguridad, nivel de expresiones, nivel de ingresos, calidad de las actividades, nivel de formación, prestigio y valoración. García (2011) propone cuatro perspectivas para su estudio: perceptivo, estructural, funcionalista y empírico. Ucrós y Gamboa (2010) señalan que para el estudio del clima organizacional es necesario considerar tres factores: psicológicos individuales, grupales, y organizacionales; con una visión integral de todos estos factores. García (2011) señala tres enfoques: subjetivo, objetivo, $\mathrm{y}$ de síntesis en el estudio del clima organizacional.

Baguer (2009) plantea que
independientemente del tipo de


organización, hay parámetros que se deben incluir en todo estudio de clima organizacional, tales como: satisfacción en el puesto de trabajo, trato personal y ambiente de trabajo, carrera profesional y promoción, reconocimiento del trabajo, estructura adecuada de la organización, delegación del trabajo, formación, comunicación y grado de confianza, condiciones de trabajo y seguridad, política medioambiental de la organización, calidad del liderazgo, conciliación del trabajo con la vida familiar, prestaciones de tipo social y factores motivadores.

Con estos antecedentes los autores de la presente investigación analizan el clima organizacional en dos ejes: a través de las percepciones individuales y grupales (enfoque perceptual); y como una manifestación de la estructura de la organización, es decir, el clima se forma porque los miembros de una organización están expuestos a unas mismas estructuras organizacionales particulares (enfoque estructural).

\section{Metodología}

Esta investigación cuantitativa se llevó a cabo entre marzo y abril del 2019. Se analizó la información de la base de datos proporcionada por la página web de la CANFAC. Se estableció que de las 37 empresas socias a nivel nacional, 19 corresponden a la provincia de Tungurahua (51.35\%). Se recolectaron 478 cuestionarios de trabajadores de 12 organizaciones $(63.16 \%)$ asociadas a la CANFAC. Las siete organizaciones restantes no entraron en estudio por ser empresas dedicadas a la comercialización y no a la producción.

El cuestionario de 17 preguntas, estructurado en 27 ítems con cuatro alternativas de respuesta (1: nunca, 2: rara vez, 3: casi siempre, 4 : siempre) en la escala de Likert fue validado por dos especialistas docentes universitarios de la carrera de psicología industrial; y por dos beneficiarios: dos directores del departamento de Talento Humano de las organizaciones encuestadas, que no formaron parte de los encuestados. Para medir el tiempo de aplicación y la efectividad del instrumento se realizó una prueba piloto que consistió en administrar el instrumento a una pequeña muestra para probar su pertinencia y relevancia (Hernández, et al. 2014) en una de las empresas asociadas a la CANFAC. Se obtuvo un alfa de Cronbach de 0,85. El valor del determinante de la matriz de correlaciones es 0,00 , lo que evidenció que las variables están linealmente relacionadas y que el modelo factorial es pertinente para el análisis de las variables. Se obtuvo un índice KMO de 0,902 , valor que confirmó que es adecuado el uso del análisis factorial en esta investigación. La prueba de esfericidad de Bartlett presentó una significancia de 0,00 , que reveló la existencia de correlaciones significativas entre las variables y reafirmó la pertinencia del modelo factorial en esta investigación. La diagonal de la matriz de correlación anti-imagen presentó coeficientes superiores a 0,70 , sólo las preguntas 15 y 24 tuvieron un valor inferior a $\quad 0,70 \quad(0,669 \quad$ y $\quad 0,677$ 
respectivamente), mostrando que las preguntas son adecuadas para medir las variables. La varianza total explicada señaló que los 5 factores explican un $49,76 \%$ de la varianza de los datos originales. Finalmente, se observó que la matriz de componentes rotados confirma que los cinco factores están medidos por todas las preguntas del cuestionario.

Este trabajo tiene un alcance descriptivo y se describieron las dimensiones que conforman el $\mathrm{CO}$ de estas industrias.

\section{Resultados}

De los 478 encuestados: 442 son hombres, y 36 mujeres; 407 son del nivel operativo, y 71 del administrativo; 189 tienen educación primaria, 220 secundaria, y 69 universitaria; 250 encuestados están entre 18 y 30 años, 142 entre 31 y 40 años, 61 entre 41 y 50 años, y 25 entre 51 y 66 años. En la Tabla 1 se exhiben las medias obtenidas de las cinco dimensiones:

Tabla 1.

Medias de las cinco dimensiones

\begin{tabular}{lc}
\hline \multicolumn{1}{c}{ Dimensión } & Media aritmética \\
\hline $\begin{array}{l}\text { Condiciones de } \\
\text { trabajo }\end{array}$ & 2.69 \\
Compensaciones & 2.90 \\
Comunicación & 3.00 \\
Liderazgo & 3.16 \\
Motivación & 3.32
\end{tabular}

Fuente: Elaboración propia.

Al analizar las respuestas correspondientes a las preguntas que conforman las cinco dimensiones, se observa que no existen valores extremos que precisen un análisis específico de cada una de ellas y/o por dimensión. Una interpretación general de los resultados, con base en las medias obtenidas en las cinco dimensiones, indican que los encuestados se sienten motivados en el trabajo, hay una comunicación medianamente fluida, un estilo de liderazgo directivo -alto interés en la ejecución de la tarea y bajo en el relacionamiento-, las condiciones de trabajo y compensaciones son percibidas como satisfactorias.

A continuación, la tabla 2 describe los porcentajes respectivos de cada pregunta de las cinco dimensiones estudiadas en la investigación. Se observa que las respuestas correspondientes a la dimensión Motivación indican que el $71.80 \%$ de los encuestados realizan las tareas con agrado, y el $63.20 \%$ se sienten motivados a asistir diariamente al trabajo, aunque los mejores momentos no siempre lo pasan en el trabajo, ni tampoco se sienten siempre valorados por los trabajos bien hechos; mayormente existe una sensación de gratificación respecto al trabajo que realizan. Hay pocos encuestados que realizan las tareas con total desagrado (el porcentaje de la opción nunca a la primera pregunta es $0.60 \%)$.

En cuanto a la dimensión Comunicación, los datos recopilados indican que la comunicación con el jefe y con los compañeros de trabajo mayoritariamente es adecuada. Así también la comunicación de logros y dificultades de la empresa al personal, aunque no siempre, es adecuada. El jefe 
Tabla 2.

Porcentajes comparativos de las 27 preguntas

\begin{tabular}{|c|c|c|c|c|}
\hline Preguntas & $\begin{array}{c}\text { Siempre } \\
4 \\
\text { Porcentaje }\end{array}$ & $\begin{array}{c}\text { Casi siempre } \\
3 \\
\text { Porcentaje }\end{array}$ & $\begin{array}{c}\text { Rara vez } \\
2 \\
\text { Porcentaje }\end{array}$ & $\begin{array}{c}\text { Nunca } \\
1 \\
\text { Porcentaje }\end{array}$ \\
\hline \multicolumn{5}{|c|}{ Dimensión: Motivación } \\
\hline 1. ¿Realiza su tarea con agrado? & 71.80 & 26.40 & 1.30 & 0.60 \\
\hline 2. ¿Se siente motivado por asistir diariamente a su trabajo? & 63.20 & 29.70 & 5.60 & 1.50 \\
\hline 3. ¿Los mejores momentos los pasa en el trabajo? & 24.10 & 54.80 & 18080 & 2.30 \\
\hline 4. ¿Se siente valorado por los trabajos bien hechos? & 32.00 & 41.20 & 21.10 & 5.60 \\
\hline 5. ¿El trabajo que realiza es gratificante para usted ? & 48.50 & 40.60 & 9.00 & 1.90 \\
\hline \multicolumn{5}{|c|}{ Dimensión: Comunicación } \\
\hline 6. ¿Se comunica adecuadamente con su jefe? & 42.50 & 37.20 & 17.20 & 3.10 \\
\hline $\begin{array}{l}\text { 7. ¿Se comunica adecuadamente con sus compañeros de } \\
\text { trabajo? }\end{array}$ & 47.90 & 40.40 & 10.50 & 1.30 \\
\hline $\begin{array}{l}\text { 8. ¿Le comunican sobre los logros y dificultades de la } \\
\text { empresa? }\end{array}$ & 35.80 & 34.70 & 22.60 & 6.90 \\
\hline 9. ¿Recibe toda la información para realizar su trabajo? & 43.10 & 37.90 & 16.10 & 2.90 \\
\hline 10. ¿Su jefe conoce sus problemas? & 15.10 & 27.00 & 35.80 & 22.20 \\
\hline 11. ¿En la empresa le escuchan sus ideas y sugerencias? & 23.40 & 46.20 & 25.30 & 5.00 \\
\hline \multicolumn{5}{|c|}{ Dimensión: Compensaciones } \\
\hline 12. ¿Es reconocido su aporte cuando se logran las metas? & 21.30 & 37.20 & 29.50 & 11.90 \\
\hline 13. ¿Son satisfactorios los beneficios y recompensas que & & & & \\
\hline recibe? & 23.40 & 38.30 & 27.00 & 11.30 \\
\hline 14. ¿Considera que sus ingresos son justos y equitativos? & 29.10 & 44.60 & 18.60 & 7.70 \\
\hline 15. ¿Es posible que sus ingresos mejoren por su trabajo? & 42.50 & 40.20 & 15.10 & 2.30 \\
\hline 16. ¿El salario le permite satisfacer sus necesidades? & 22.60 & 51.50 & 19.50 & 6.50 \\
\hline \multicolumn{5}{|c|}{ Dimensión: Liderazgo } \\
\hline 17. ¿Las órdenes que recibe son actibles de cumplir? & 42.10 & 49.60 & 7.50 & 0.60 \\
\hline $\begin{array}{l}\text { 18. ¿Sus superiores mantienen una buena relación con } \\
\text { usted ? }\end{array}$ & 41.40 & 42.10 & 12.80 & 3.80 \\
\hline 19. ¿Le dan a conocer los reglamentos de la organización? & 52.50 & 28.20 & 15.90 & 3.30 \\
\hline 20. ¿La empresa le brinda oportunidades de capacitarse? & 34.30 & 30.10 & 24.30 & 11.30 \\
\hline 21. ¿Cuándo tiene problemas recibe apoyo de la empresa? & 43.70 & 31.00 & 17.40 & 7.90 \\
\hline \multicolumn{5}{|c|}{ Dimensión: Condiciones de trabajo } \\
\hline 22. ¿Dispone de suficiente espacio para realizar su trabajo? & 44.80 & 41.00 & 12.60 & 1.70 \\
\hline $\begin{array}{l}\text { 23. ¿Corre riesgo su integridad física por el manejo de } \\
\text { equipos y herramientas? }\end{array}$ & 20.30 & 31.20 & 32.60 & 15.90 \\
\hline $\begin{array}{l}\text { 24. ¿Le causan molestias los ruidos y olores de los } \\
\text { productos que se emplean en la empresa? }\end{array}$ & 26.40 & 28.50 & 33.30 & 11.90 \\
\hline $\begin{array}{l}\text { 25. ¿Es adecuada la temperatura e iluminación en su lugar } \\
\text { de trabajo? }\end{array}$ & 37.70 & 37.00 & 19.70 & 5.60 \\
\hline $\begin{array}{l}\text { 26. ¿Se ha enfermado por las condiciones en las realiza su } \\
\text { trabajo? }\end{array}$ & 12.80 & 25.50 & 39.70 & 22.00 \\
\hline 27. ¿Le causa estrés su trabajo? & 14.90 & 20.50 & 38.70 & 25.90 \\
\hline
\end{tabular}

Fuente: Elaboración propia. 
mayoritariamente (58\%) desconoce sobre los problemas que tiene el personal, y casi siempre las ideas y sugerencias del personal son escuchadas.

Con relación a la dimensión Compensaciones, las respuestas indican que existe una sensación dividida en cuanto a los beneficios y recompensas recibidas por los encuestados. Se observa que los encuestados consideran que sus ingresos posiblemente mejoren por el trabajo siempre y casi siempre. En un porcentaje mayoritario $(82.70 \%)$, señalan al trabajo como una vía posible para mejorar los ingresos, y los beneficios y recompensas son calificados como satisfactorios.

Respecto a la dimensión Liderazgo, los resultados indican que existe un alto porcentaje de empleados que consideran factible cumplir las órdenes recibidas, ya que los porcentajes de siempre y casi siempre así lo confirman. De todo el universo encuestado, un bajo porcentaje $(0.60 \%)$ respondió con la opción nunca. Existe una mayoritaria sensación de considerar buena la relación con los superiores; los puntajes altos de las opciones siempre $(41.40 \%)$ y casi siempre $(42.10 \%)$ lo corroboran.

Los empleados en su mayoría (52.50\%) conocen sobre los reglamentos de la organización. El análisis global de este factor indica que existe una buena relación jefe-empleado, los empleados reciben información adecuada sobre los reglamentos de la organización y perciben como realizables sus tareas. Los empleados sienten en un porcentaje ligeramente mayoritario, apoyo de la empresa al enfrentar problemas.

En cuanto a la última dimensión: Condiciones de trabajo, los datos recolectados indican que el espacio físico es percibido por el $85.80 \%$ de los encuestados como suficientemente adecuado para la realización del trabajo. Existe un porcentaje mayoritario de empleados $(74.70 \%)$ para quienes la temperatura e iluminación en el sitio de trabajo es adecuada. Casi la mitad de los encuestados (existencia de valores cercanos al 50\%) sienten que corre peligro su integridad física y les molesta los ruidos y olores. Un porcentaje minoritario señala que se ha enfermado por las condiciones laborales y que les ha generado estrés.

Se encontró que las mujeres desarrollan una mejor comunicación tanto vertical como horizontal, lo cual incide en que puedan tener una percepción más positiva del ambiente. De manera similar en un estudio realizado por Lazo y Lazo (2018) se obtuvo que las mujeres tienen una actitud más favorable que los hombres con respecto al clima laboral. Sin embargo, las mujeres están menos motivadas que los hombres para asistir diariamente al trabajo. Por otra parte, las mujeres perciben mayor satisfacción que los hombres con respecto a sus ingresos y recompensas. También las mujeres poseen una mejor percepción de sus condiciones de trabajo que los hombres, los cuales, además, se enferman más por causas relacionadas con el trabajo; sin embargo, las mujeres son más vulnerables al estrés. En cuanto 
al liderazgo, las mujeres tienen mejores relaciones que los hombres con sus superiores y también las mujeres reciben más apoyo que los hombres cuando tienen problemas.

De acuerdo con Lazo y Lazo (2018), a mayor edad menor involucramiento laboral y viceversa. En la presente investigación, a mayor edad, mayor disfrute del trabajo y mayor la gratificación por sentirse valorados $\mathrm{y}$ reconocidos. No existen diferencias en cuanto a la edad para sentir motivación para asistir diariamente al trabajo ni para sentirse escuchados en cuanto a ideas y sugerencias. Los trabajadores a partir de los 41 años tienen una percepción más positiva en cuanto a la justeza y equidad de sus ingresos. También a mayor edad, mejores posibilidades de satisfacer necesidades con el salario, lo cual también se aprecia en los trabajadores más jóvenes. También se encontró una mejor percepción del apoyo de la empresa cuando tienen problemas en los trabajadores mayores de 51 años, así como una mejor percepción de sus oportunidades de capacitación.

Los colaboradores en cargos administrativos se sienten más gratificados en su trabajo que los operarios; sin embargo, los operarios manifiestan mayor motivación que los administrativos por asistir diariamente al trabajo. Ambos grupos de cargos consideran gratificante el trabajo que realizan. En contraste, un estudio realizado por Jiménez - Bonilla y Jiménez - Bonilla (2016) encontró que en especial los administrativos de una empresa de consumo masivo presentaron insatisfacción con el liderazgo, la motivación y la participación, variables también abordadas en el estudio como componentes del CO. En cuanto a Comunicación los resultados de los administrativos son ligeramente más favorables que laos de los operarios, ya que los últimos no siempre reciben comunicaciones oportunas sobre dificultades o logros de la empresa; y tampoco consideran que sus problemas sean conocidos por sus superiores con mucha frecuencia.

Con respecto a las condiciones de trabajo, hay mayor percepción de riesgo de la integridad física en los operadores que en los administrativos. Hay mayor satisfacción con los aspectos de temperatura e iluminación en los administrativos que en los operadores. Los operadores se enferman más que los administrativos por las condiciones en que realizan su trabajo. Este resultado es similar al obtenido por Carballo (2015) en un estudio realizado en personal operativo federal del Programa Caravanas de la Salud en Tabasco, México, para determinar la relación entre clima laboral y satisfacción, donde el personal operativo también manifestó insatisfacción con su infraestructura y equipamiento, así como con el ambiente físico para realizar el trabajo, afectándose con esto el clima laboral ya que existe correlación entre ambas variables.

En cuanto a la motivación por el trabajo, el nivel de instrucción no marca diferencias para el agrado con el cual se realiza el trabajo, el sentirse bien, 
considerar gratificante lo que hacen y asistir diariamente al trabajo. Sin embargo, sí se observa una ligera diferencia en la percepción de la valoración recibida con respecto al trabajo realizado, siendo el personal con nivel de instrucción primaria el que tiene una mejor percepción al respecto; mientras que los de nivel secundaria y universitario no siempre se sienten valorados por los trabajos bien hechos. En general todo el personal, independientemente del nivel de instrucción, se siente mayormente motivado a asistir diariamente al trabajo.

A pesar de que en el nivel de instrucción no aparecen diferencias relacionadas con la motivación, sí las hay con respecto a la compensación, siendo los universitarios los que perciben de manera más favorable el reconocimiento por su trabajo, por los beneficios y recompensas, la justeza y equidad de sus ingresos con respecto al trabajo que desempeñan, la posibilidad de resolver sus problemas con el salario y se manifiestan más optimistas con respecto a sus posibilidades de mejorar sus ingresos. Con respecto a las condiciones de trabajo y el nivel de instrucción, en general los universitarios vuelven a tener una percepción más favorable con relación al espacio, los olores, ruidos, la temperatura y la iluminación. Los trabajadores de niveles primario $\mathrm{y}$ secundario suelen tener una percepción menos favorable con respecto a los aspectos anteriores $\mathrm{y}$, además, suelen enfermarse más que los universitarios por aspectos relacionados con el trabajo. Sin embargo, los trabajadores universitarios refieren mayor estrés causado por el trabajo. En cuanto al liderazgo, los trabajadores universitarios tienen una mejor percepción de buena relación con los superiores y poseen mejores conocimientos de los reglamentos, lo cual favorece la predisposición para acatar órdenes. La capacitación constituye un aspecto débil en la empresa, siendo los universitarios los que perciben mayor afectación.

\section{Conclusiones}

En esta investigación se evidencia que las mujeres desarrollan una mejor comunicación tanto vertical como horizontal, lo cual incide en que puedan tener una percepción más positiva del ambiente. De manera similar en un estudio realizado por Lazo y Lazo (2018) se obtuvo que las mujeres tienen una actitud más favorable que los hombres con respecto al clima laboral. Sin embargo, las mujeres están menos motivadas que los hombres para asistir diariamente al trabajo, siendo quizás la razón por la que también manifiestan mayor vulnerabilidad al estrés en comparación con los hombres. Por otra parte, las mujeres perciben mayor satisfacción que los hombres con respecto a sus ingresos y recompensas. También las mujeres poseen una mejor percepción de sus condiciones de trabajo que los hombres, los cuales, además, se enferman físicamente más por causas relacionadas con el trabajo.

En cuanto al liderazgo, las mujeres tienen mejores relaciones que los hombres con sus superiores. Este 
resultado es congruente con lo encontrado por Omar y Uribe - Delgado (2011) en una investigación sobre la vinculación del alocentrismo- idiocentrismo con las percepciones de justicia en el trabajo, en la cual las mujeres de la investigación resultaron ser más alocéntricas que los hombres, siendo sus características las de preocuparse más por la armonía grupal, cumplir normas de convivencia y sacrificarse por el logro de objetivos comunes; lo cual guarda relación positiva con la percepción de justicia interaccional.

Una investigación de la Universidad de Murcia, España, realizada en el sector turístico, plantea que "la mujer está mejor preparada para la cooperación y empatía, la comunicación, el trabajo en equipo, mayor flexibilidad y adaptabilidad a cambios repentinos, orientación al grupo, visión global, perspectiva consensual y democrática del poder" (Fernández y Martínez, 2010, p. 148). Debido a ello las mujeres, a partir de su propio desempeño, establecen relaciones más positivas que los hombres, lo cual se revierte en su propia percepción de mejores relaciones en el ámbito laboral.

Por otra parte, hay investigaciones como la de Gómez y Sánchez (2009) donde se plantea que "rasgos psicológicos, como la expresividad emocional o las habilidades comunicativas, y competencias directivas, como la capacidad de síntesis o de saber negociar ante una situación conflictiva, pueden estar muy acusadas en una mujer y muy poco en otra" (p.132). Con esta idea el género no tendría influencia en la forma en que suceden las interacciones humanas dentro de la organización y, por tanto, sería irrelevante su análisis en función de este dato sociodemográfico.

Se deben revisar los mecanismos de comunicación interna establecidos, aun cuando la comunicación en general se ubica en un coeficiente de 3,00 , se la podría mejorar con énfasis en aquellos que permitan que las personas comuniquen de forma ascendente los problemas de índole personal que les pueden afectar en el desempeño del trabajo, ya que se manifiesta que los jefes rara vez conocen los problemas del personal. Lo operarios señalan que la comunicación descendente fluye solamente para compartir información relativa a las tareas a cumplir. Este problema puede afectar la integración de los colaboradores con la empresa, y por ende, su sentido de pertenencia.

Otro aspecto sensible es la compensación, la cual no satisface al grupo etario entre 18 y 40 años, siendo éstas las edades en que probablemente las personas tienen cargas familiares que mantener económicamente. Este es un aspecto que puede provocar una elevación en el índice de rotación externa, debido a la búsqueda de mejores oportunidades.

La capacitación debe ser fortalecida en el personal entre 41 y 50 años, pues ellos demandan mejores oportunidades de formación. La perspectiva andragógica sugiere cambiar de paradigma en la forma de concebir el aprendizaje en general, y la capacitación-formación 
laboral específicamente, es necesario pasar de un enfoque de enseñanzaaprendizaje a uno de actividad enfocado en la orientación-aprendizaje adulto, es decir, dejar de lado el criterio individualista y pasar a una visión de discurso colectivo (Rivas, 2012).

Con respecto a las condiciones de trabajo, las empresas deben adoptar las medidas necesarias de seguridad y salud en el trabajo, de forma que no existan incidencias de esta índole, los operarios, mayormente entre los 31 y 40 años, debe ser el grupo donde focalizar la atención, ya que es el conjunto que mayormente se enferma por las condiciones de trabajo.

\section{Referencias}

Alcover de la Hera, C., Martínez, D., Rodríguez, F., y Domínguez, R. (2004). Introducción a la psicología del trabajo. Madrid, España: Editorial McGraw-Hill Interamericana

Baguer, A. (2009). Dirección de Personas. Un timón en la tormenta. 2da Ed. España: Ediciones Díaz de Santos.

Bamel, U., Rangnekar, S., Stokes, P., y Rastogi, R. (2015). Managerial effectiveness: An Indian experience. Journal of Management Development, 34(2), 202-225.doi.org/10.1108/jmd-10-2012-0129

Barzola-Zambrano, I., Barzola-Véliz, V., y FloresBarzola, W. (2017) Factores del clima laboral que influyen en el rendimiento de los trabajadores del sector público en el Ecuador. Revista cientifica. Dominio de las ciencias, 3(3), 917-937.

Cardona, D. y Zambrano, R. (2014). Revisión de instrumentos de evaluación de clima organizacional. Estudios Gerenciales, 30, 184-189. doi.org/10.1016/j.estger.2014.04.007
Carballo, A. (2015). Clima organizacional y satisfacción laboral en el personal operativo federal del programa Caravanas de la salud en Tabasco, México. Hitos de Ciencias Económico Administrativas. 21(59), 43 - 51. Recuperado de http://revistas.ujat.mx/index.php/hitos/art icle/view/1011

Davis, K. y Newstrom, J. (2003). El comportamiento humano en el trabajo (Sexta edición ed.) México: McGraw-Hill.

Echeverri, D. y Cruz, R. (2014). Revisión de instrumentos de evaluación de clima organizacional. Estudios Gerenciales, 30(131), 184-189. doi.org/10.1016/j.estg er.2014.04.007

Guízar, R. (2013). Desarrollo organizacional: principios y aplicaciones. México D.F., México: Mc Graw Hill

Fernández, M. y Martínez, L. (2010). Participación de las mujeres en las empresas turísticas privadas y comunitarias de bahías de Huatulco, México. ¿Hacia un cambio en el rol de género? Cuadernos de Turismo. 26 (2010), 129 - 151. Recuperado de: https://revistas.um.es/turismo/article/vie w/116311

Flores, M., Vega, A., y Chávez, E. (2015). El clima organizacional como factor de competitividad en las franquicias de comida rápida en Tijuana, B.C., México. Revista Internacional Administración y Finanzas, 8(5), 25-44.

Furnham, A. (2006). Psicología organizacional. (G. Ortega, Trad.) México, D.F., México: Alfaomega.

Gamboa-León, L. (2014). Clima laboral de la empresa Bombonería Di Perugia. Revista de ciencias empresariales de la Universidad San Martín de Porres, 5(1), 3-12.

García, M. (2011). Clima Organizacional y su Diagnóstico: Una aproximación Conceptual. 
Cuadernos de Administración, 42. 43-61. doi.org/10.25100/cdea. v25i42.413

Gómez, J. y Sánchez, M. (2009). La participación de la muj er en los consejos de administración de empresas del Ibex-35. Revista de Humanidades. 16(2009), 105 139. Recuperado de:https://dialnet.unirioja. es/servlet/articulo? codigo $=3301218$

Iglesias, A. y Sánchez, Z. (2015). Generalidades del clima organizacional. Medisur, 13(3), 455-457.

Jácome, L. (2017). Canfac Ecuador. Recuperado de: http://canfacecuador.com/index.php/bonu s-page

Jiménez - Bonilla, D. y Jiménez - Bonilla, E. (2016). Clima laboral y su incidencia en la satisfacción de los trabajadores de una empresa de consumo masivo. Revista Ciencia UNEMI. 9(18), 26 - 34.

Recuperado de: http://ojs.unemi.edu.ec/in dex.php/cienciaunemi/article/view/301

Lazo, M. y Lazo, A. (2018). Clima laboral y vulnerabilidad al estrés en trabajadores de una empresa de industria textil. Revista de Psicología. 8(2), 61 - 79. Recuperado de:http://ucsp.edu.pe/investigacion/psicol ogia/wp-content/uploads/2019/04/Rev-ps icol-UCSP-2018-2.pdf\#page $=63$

Lewin, K. (1988). La teoría de campo en las ciencias sociales. España, Barcelona: Paidós.

Likert, R. (2007). New patterns of management. USA, Michigan: McGraw-Hill.

Marchant, L. (2005). Actualizaciones para el desarrollo organizacional. Primer seminario Viña del Mar. Chile.

McGregor, D. (1960). The human side of Enterprise. USA, New York: McGraw-Hill.

Omar, A. y Uribe-Delgado, H. (2011). Vinculaciones del alocentrismo-idiocentrismo con las percepciones de justicia en el trabajo.
Revista Interamericana de Psicología Ocupacional. 30(1), 5- 20. Recuperado $\mathrm{d}$

http://revista.cincel.com.co/index.php/RP $\mathrm{O} /$ article/view/111

Rivas, E. (2012). Andragogía: Educación de adultos, ¿Ciencia o disciplina?: El adulto como persona. España: Editorial Académica Española.

Romero, M. (2008). Psicología institucional. Quito, Ecuador: CODEU.

Salazar, J., Guerrero, J., Machado, Y., y Cañedo, R. (2009). Clima y cultura organizacional: dos componentes esenciales en la productividad laboral. ACIMED, 20 (4), 67-75.

Silva, M., Santos, J., Rodríguez, E., y Hernando, C. (2008). Las relaciones humanas en la empresa (Primera ed.). España: Paraninfo.

Ucrós, M. y Gamboa, T. (2010). Clima organizacional: discusión de diferentes enfoques teóricos. Visión gerencial, 1, 179-190. Recuperado de: http://www.redalyc.org/ articulo.oa?id $=46554588$ 